\title{
Positive psychology interventions to improve well-being and symptoms in people on the schizophrenia spectrum: a systematic review and meta-analysis
}

\author{
Isabela Pina, ${ }^{1}$ iD Catarina de M. Braga, ${ }^{2}$ iD Túlio F.R. de Oliveira, ${ }^{3}$ Camila N. de Santana, ${ }^{1}$ Rodrigo \\ C. Marques, ${ }^{2}$ iD Leonardo Machado $^{2}$ iD \\ ${ }^{1}$ Hospital das Clínicas, Universidade Federal de Pernambuco (UFPE), Recife, PE, Brazil. ${ }^{2}$ Programa de Pós-Graduação em Neuropsiquiatria e \\ Ciências do Comportamento, UFPE, Recife, PE, Brazil. ${ }^{3}$ Universidade de Pernambuco (UPE), Recife, PE, Brazil.
}

\begin{abstract}
Objective: Positive psychology interventions (PPIs) incorporate principles of personal strengths with the view that mental health recovery transcends symptom relief. Severe psychiatric conditions, such as schizophrenia, may benefit from such interventions. This study aims to gather the current evidence on the impact of PPIs on increasing well-being in patients on the schizophrenia spectrum and assess reductions in negative or positive symptoms.

Methods: A systematic review of PPI studies with schizophrenia-spectrum patients was carried out following PRISMA recommendations. The PubMed, PsycINFO, Web of Science, and Scopus databases were searched for relevant publications in order to understand the possible effects of these interventions on well-being measures and psychotic symptoms in this population.

Results: Nine studies (four controlled) were included. Meta-analysis of the controlled studies showed a significant effect $(p=0.04)$ for improvement of well-being $(Z=2.01)$. Overall, the reviewed evidence suggests well-being improvement. The effect on reduction of negative symptoms was unclear.

Conclusion: Used as an adjunctive therapy, PPIs appear to be a promising resource for patients on the schizophrenia spectrum, with possible effects on well-being and symptom reduction.
\end{abstract}

Keywords: Schizophrenia; positive psychology; well-being; positive psychiatry

\section{Introduction}

In recent decades, psychiatry has been concerned not only with the remission of symptoms but also with quality of life, a return to previous functionality, and well-being - a paradigm shift which has transformed outcomes in research on mental illness. ${ }^{1}$ The concept of recovery has also generated increasing interest. Recovery may be understood as a way of living a satisfying, contributing, realistically hopeful life, while developing meaning and purpose in life beyond the limiting and stigmatizing effects of mental illness. ${ }^{2}$ Recovery from mental disorder may be improved if, in addition to treating symptoms, interventions emphasize the development of personal resources and skills, such as identifying strengths and building a positive identity.

Positive psychology interventions (PPIs), which incorporate principles based on personal strengths, are aligned with the view that mental health recovery transcends symptom relief to include experiencing positive emotions, satisfaction, and purpose, thus promoting well-being. PPIs may also be applied to strengthen personal and social

Correspondence: Isabela de Fátima Pina de Almeida, Rua Dr. Virgínio Marques, 285/103, Bloco A, Iputinga, CEP 50731-330, Recife, PE, Brazil.

E-mail: bela_pina@ hotmail.com

Submitted May 23 2020, accepted Aug 18 2020, Epub Dec 182020. relationships; many include exercises such as the activeconstructivist response, which aims to improve well-being within individual relationships in contexts of listening to positive news communicated by others. A strategy that promotes positive affect and facilitates the use of positive interpersonal skills may plausibly improve the quality of relationships. $^{3}$

PPIs started as interventions for people without a formal psychiatric diagnosis, and were further developed for use in patients with mood disorders. ${ }^{4}$ Starting with the WELLFOCUS positive psychotherapy (PPT) initiative, modified protocols for psychotic patients have been in development. ${ }^{5}$ Since then, several other protocols for psychotic patients have used different combinations of PPIs, such as the French Positive Emotions Program for Schizophrenia (PEPS). ${ }^{6}$

Schizophrenia is a complex behavioral and cognitive syndrome that severely impairs individual function and deeply impacts the surrounding social environment. It is characterized by a variable clinical presentation, but usually features positive symptoms (hallucinations, delusions), negative symptoms (emotional blunting, social

How to cite this article: Pina I, Braga CM, de Oliveira TFR, de Santana CN, Marques RC, Machado L. Positive psychology interventions to improve well-being and symptoms in people on the schizophrenia spectrum: a systematic review and meta-analysis. Braz J Psychiatry. 2021;43:430-437. http://dx.doi.org/10.1590/15164446-2020-1164 
withdrawal), and cognitive impairment (inattention, executive dysfunction). ${ }^{7}$ Some studies involving patients with schizophrenia showed that baseline quality of life and subjective well-being were able to significantly predict symptom remission and adherence to drug treatment. Therefore, concern for the well-being and recovery of these patients has become fundamental and one of the main outcomes to be achieved. ${ }^{8}$

The present study aimed to conduct a systematic review and meta-analysis of the current evidence on the impact of PPIs in increasing the well-being of patients on the schizophrenia spectrum (primary outcome), in addition to assessing the impact of these interventions on reduction of negative and/or positive symptoms of schizophrenia (secondary outcomes).

\section{Methods}

\section{Search strategy}

Following the PRISMA protocol, ${ }^{9}$ a structured search and systematic review of the literature was carried out independently by two authors (IP and RM), in July 2019. The PubMed, PsycINFO, Web of Science, and Scopus databases were searched. No language or date filters were applied. The search terms used were: "Schizophrenia spectrum and other psychotic disorders" or "Schizophrenia" or "Psychosis" or "Psychotic" combined with "Positive Psychology" or "Positive Psychiatry" or "Positive Psychotherapy." All results retrieved by the above combination were surveyed.

This review was submitted to PROSPERO (registration pending, ID 156064). A handsearch of the reference lists of included articles and of reviews related to the topic found during the database search yielded no additional results.

\section{Study selection}

\section{Inclusion and exclusion criteria}

Disagreements were discussed with a third researcher (LM) until a consensus was reached. Eleven articles were selected for full-text reading and checked against the inclusion criteria, namely:

1. Clinical intervention studies using positive psychology in general or PPT in particular, and with positive psychology pre-intervention and post-intervention outcome procedures measured by scales that assess well-being directly or indirectly (through related constructs to the concept of well-being) (primary outcome) and an improvement in positive and/or negative symptoms of schizophrenia using symptom scales (secondary outcome);

2. Studies with a sample formed of patients diagnosed with schizophrenia, schizoaffective disorder, or delusional disorder. Diagnosis should follow the ICD-10 (F20, F22 or F25), ${ }^{10}$ DSM-IV-TR ${ }^{11}$ or DSM- $5^{12}$ criteria and be performed by a trained psychiatrist. Studies that assessed populations at ultra-high risk for psychosis (UHR) were excluded, as this is not a psychiatric diagnosis, but a risk classification.
Due to the wide variety of scales known to be used in the literature for assessing well-being, ${ }^{13}$ no specific scales were selected beforehand; this allowed us to carry out a more wide-ranging review.

\section{Data extraction}

Data extraction and quality assessment were performed by the two reviewers and checked once again by a third researcher. Disagreements were assessed jointly by all researchers. Data were collected on study characteristics (country of origin, study design, quality analysis), sample (diagnostic criteria, patient characteristics, sample size), and PPI (type of intervention, timing of outcome assessment, and the instruments used to assess outcome).

\section{Quality assessment}

The quality of the included studies was assessed with a short five-item scale adapted from the Cochrane Collaboration quality assessment instrument and the Valiente et al. ${ }^{14}$ review of psychotherapy interventions: 1) randomization sequence; 2) blinding of outcome assessment; 3) baseline comparability (if study groups were comparable at baseline); 4) power of the analysis or an $n \geqslant 50$ (if there was an appropriate power analysis and/or at least 50 participants in the analysis); 5) imputation of missing data or analysis by intention to treat. Each criterion was classified as 0 (study does not meet the criteria) or 1 (study meets the criteria). The quality of the studies was scored from 1 to 5 . Arbitrarily, studies with a score of 4 or 5 were considered of high quality.

\section{Data synthesis}

The quantitative data analysis was performed using Review Manager 5.3 (MacOS, Cochrane Collaboration, http://tech.cochrane.org/Revman). An inverse-variance random effects model was chosen because of the high potential for heterogeneity in the included studies. ${ }^{15}$ Study heterogeneity was investigated using the $\chi^{2}$ test of homogeneity $(p<0.05)$ and $I^{2}$ statistic. $^{16}$

All outcomes were presented as continuous variables in the form of mean and standard deviation. A forest plot was generated considering a 95\% confidence interval $(95 \% \mathrm{Cl})$ and the final standardized mean difference between groups. Data used in the meta-analysis were restricted to the first post-intervention measures; additional follow-up measures were not included.

For studies with a high risk of bias, we performed a sensitivity analysis excluding articles that scored lower than 3 on the quality assessment tool.

\section{Results}

The quality assessments for each study are presented in Table 1.

The main characteristics of the reviewed studies are presented in Table 2. 
Table 1 Quality analysis of selected studies

\begin{tabular}{|c|c|c|c|c|c|c|}
\hline Study & Randomization & Blinding & Baseline & Power of analysis or $n \geqslant 50$ & Missing data & Total \\
\hline Johnson $^{17}$ & 0 & 0 & 1 & 0 & 1 & 2 \\
\hline Meyer $^{3}$ & 0 & 0 & 1 & 0 & 1 & 2 \\
\hline Favrod $^{18}$ & 0 & 0 & 1 & 0 & 0 & 1 \\
\hline Schrank $^{19}$ & 1 & 0 & 1 & 1 & 1 & 4 \\
\hline Meyer-Kalos ${ }^{20}$ & 0 & 0 & 1 & 0 & 0 & 1 \\
\hline $\mathrm{Kim}^{21}$ & 1 & 1 & 1 & 1 & 0 & 4 \\
\hline Kang $^{22}$ & 0 & 0 & 1 & 1 & 0 & 2 \\
\hline Favrod $^{6}$ & 1 & 1 & 1 & 1 & 1 & 5 \\
\hline $\operatorname{Lim}^{23}$ & 0 & 0 & 1 & 0 & 0 & 1 \\
\hline
\end{tabular}

\section{Description of studies}

A total of 378 patients were assessed. Of these, 238 received PPIs, and 140 continued treatment as usual (control group). Four studies used controlled designs..$^{6,19,21,22}$ The other studies used pre- and post-intervention assessments. In eight studies, participants were recruited from outpatient clinics and specialized centers for psychotic disorders. $^{3,6,17,19-21,23}$ In one study, patients were admitted to a psychiatric ward. ${ }^{22}$ One study assessed intervention using a smartphone app (+Connect), ${ }^{23}$ with aid and support from therapists once a week.

Two articles were excluded after full-text reading. The first did not list selected data on patients with bipolar and schizophrenia-spectrum disorders; in addition, 29 participants had already been recruited for an Early Intervention for Psychosis. The second did not use an intervention considered adequate for comparison with other studies in this review, since it did not clearly discriminate data from PPI (NAVIGATE integrated care program). ${ }^{24}$ One study was in Korean, but tables and results were presented in English, and was thus included; the full text was translated for analysis. Nine studies composed the final review (Figure 1).

Different PPI protocols were used in the studies included in this review; many were pilot studies to assess the viability and acceptance of interventions before constructing a protocol for larger groups of patients. The interventions consisted of modifications of PPTs adapted to patients with psychotic disorders. Two studies used the PPT adapted for people with schizophrenia protocol (WELLFOCUS). ${ }^{25}$ Two other studies used the PEPS protocol. ${ }^{26}$ The other individual studies used the following interventions: Positive Living, ${ }^{22}$ Individual Coping Awareness Therapy (I-CAT), ${ }^{20}$ Loving-Kindness Meditation (LKM), ${ }^{17}$ + Connect (smartphone app), ${ }^{23}$ and Mental Fitness. ${ }^{22}$

With regard to the primary outcome, two articles reported no statistically significant improvement, ${ }^{6,19}$ while the other studies reported improvement in outcome measures related to well-being.

In addition to assessing the impact of PPIs on wellbeing outcomes, most studies included outcome measures of overall symptom severity, especially negative and depressive symptoms. ${ }^{3,6,19,23}$ These assessments were made using the Positive and Negative Syndromes Scale (PANSS) ${ }^{27}$ and the Scale of Brief Psychiatric Evaluation (BPRS). ${ }^{28}$ All studies reported significant improvement on these scales, with moderate and sustained effects on follow-up.

Thus, PPI as an adjunct to drug treatment appears to be effective in increasing outcomes related to well-being and quality of life, as well as reducing negative symptoms in patients with schizophrenia spectrum disorders.

\section{Meta-analysis}

Only the four studies that used control groups and blinding strategies were included in this step. Quantitative analysis of the four controlled studies demonstrated high heterogeneity $\left(I^{2} 92 \%\right.$; chi-square 9.01$)$. The pooled effects estimate was $1.19(95 \% \mathrm{Cl} 0.29-2.09, \mathrm{p}=0.009)$, favoring the experimental group (Figure 2).

\section{Sensitivity analysis}

A sensitivity analysis was performed and a separate forest plot generated excluding the Kang et al. ${ }^{22}$ article, which reduced heterogeneity to $\mathrm{I}^{2}=78 \%$. The pooled effects estimate was $0.72(95 \% \mathrm{Cl} 0.14-1.31, \mathrm{p}=0.02)$, also favoring the intervention (Figure 3 ).

\section{Discussion}

This systematic review included nine clinical studies that used modified PPIs for patients with schizophreniaspectrum disorders. In recent years, there seems to have been a marked increase in psychiatric interest in the study of positive psychology concepts. An understanding that quality of life and psychosocial functioning depend less on physical health and more on positive psychological traits, such as resilience, optimism, social engagement and wisdom, has transformed the way in which mental illness is viewed. ${ }^{29}$

The studies in this review used protocols based mainly, but not exclusively, on positive psychology, such as WELLFOCUS, PEPS, Positive Living, I-CAT, + Connect and Mental Fitness. Therefore, there was significant variability in assessment methods, and data comparisons should be viewed with caution.

Clinical outcomes (such as negative symptoms) in schizophrenia can also be significantly modified by PPI, as demonstrated by one of the reviewed articles, which proposed a meditation protocol (LKM) with a focus on increasing the use of experiences in the present. In four of 


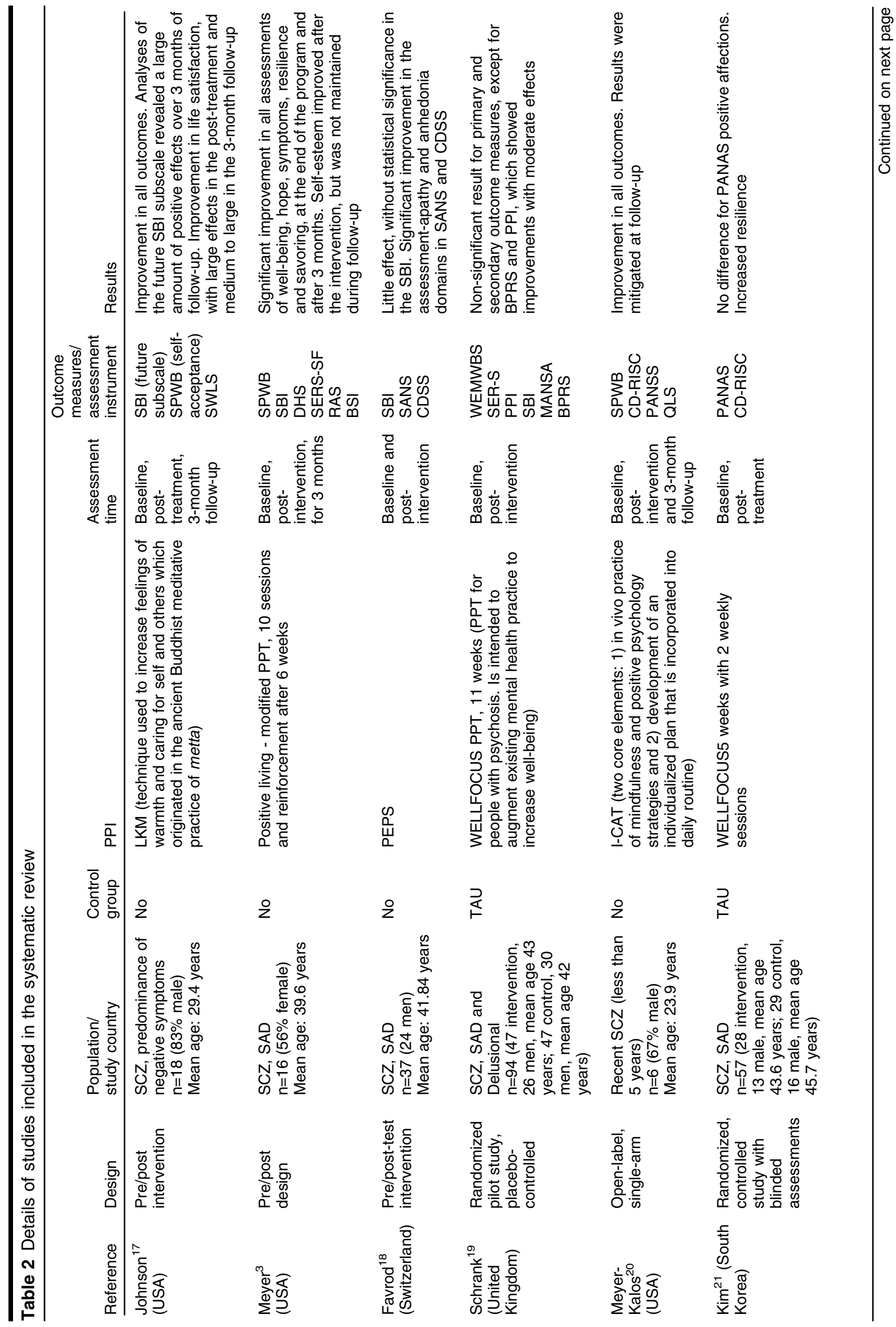




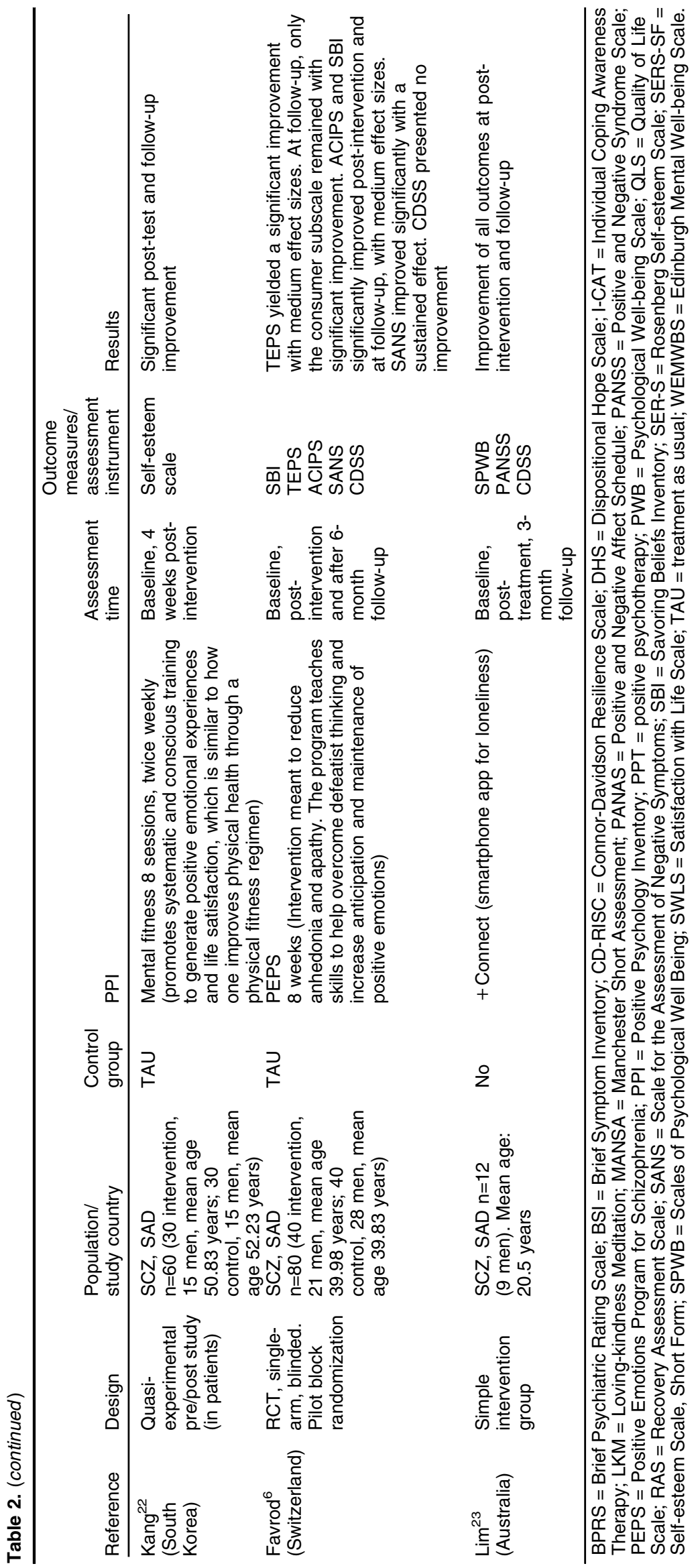



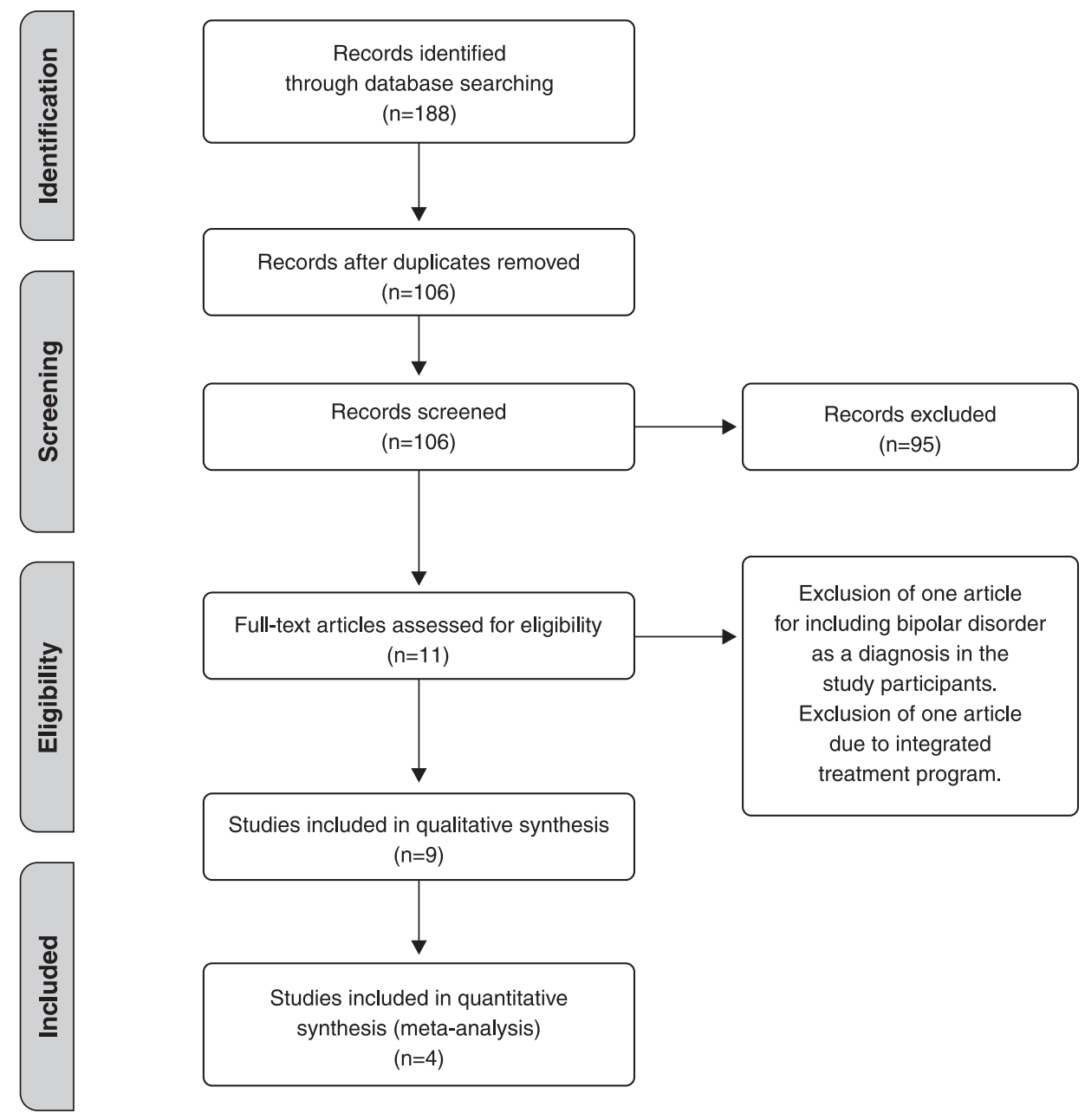

Figure 1 PRISMA flowchart for study selection.

\begin{tabular}{|c|c|c|c|c|c|c|c|c|c|c|c|c|}
\hline $\begin{array}{l}\text { Study or } \\
\text { subgroup }\end{array}$ & \multicolumn{3}{|c|}{ Experimental } & \multicolumn{3}{|c|}{ Control } & Weight & $\begin{array}{l}\text { Std. mean difference } \\
\text { IV, Random, } 95 \% \mathrm{Cl}\end{array}$ & \multicolumn{3}{|c|}{$\begin{array}{l}\text { Std. mean difference } \\
\text { IV, Random, } 95 \% \mathrm{Cl}\end{array}$} & \\
\hline Schrank 2015 & 3.36 & 0.66 & 43 & 3.24 & 0.64 & 41 & $25.8 \%$ & $0.18[-0.25,0.61]$ & & & -1 & \\
\hline Favrod 2019 & 23.03 & 5.45 & 40 & 21.78 & 6.06 & 40 & $25.7 \%$ & $0.21[-0.22,0.65]$ & & & -1 & \\
\hline Kim 2017 & 90.11 & 16.15 & 28 & 77.28 & 16.08 & 29 & $25.0 \%$ & $0.79[0.24,1.33]$ & & & -1 & \\
\hline Kang 2018 & 93.9 & 11.84 & 30 & 67.47 & 6.84 & 30 & $23.6 \%$ & $2.70[1.99,3.41]$ & & & & - - \\
\hline Total $(95 \% \mathrm{Cl})$ & & & 141 & & & 140 & $100.0 \%$ & $0.93[-0.01,1.87]$ & & & & \\
\hline \multicolumn{9}{|c|}{$\begin{array}{l}\text { Heterogeneity: } \operatorname{Tau}^{2}=0.85 ; \mathrm{Chi}^{2}=40.42, \mathrm{df}=3(p<0.00001) ;\left.\right|^{2}=93 \% \\
\text { Test for overall effect: } Z=1.95(p=0.05)\end{array}$} & -4 & -2 & 0 & 2 \\
\hline
\end{tabular}

Figure 2 Forest plot and extent of the effects of primary outcomes on well-being and quality of life. $95 \% \mathrm{Cl}=95 \%$ confidence interval; $\mathrm{df}=$ degrees of freedom; IV = inverse variance; $\mathrm{SD}=$ standard deviation.

the nine articles, there was a statistically significant reduction in the symptoms of schizophrenia assessed by PANSS and BPRS. These data seem promising, since pharmacological interventions have failed to demonstrate a major impact on the negative symptoms of schizophrenia. ${ }^{6}$

In addition to the LKM protocol, I-CAT, PEPS, and Positive Living also used some form of meditation, especially mindfulness. Meditation is associated with positive emotions, increased well-being, and use of the moment, and has proven utility in the management of psychosis. ${ }^{30,31}$ However, in the case of people on the schizophrenia spectrum, studies have shown that there are risks of adverse effects from the use of meditative practices; leading them to be considered a contraindication or making it necessary to pay more attention during 


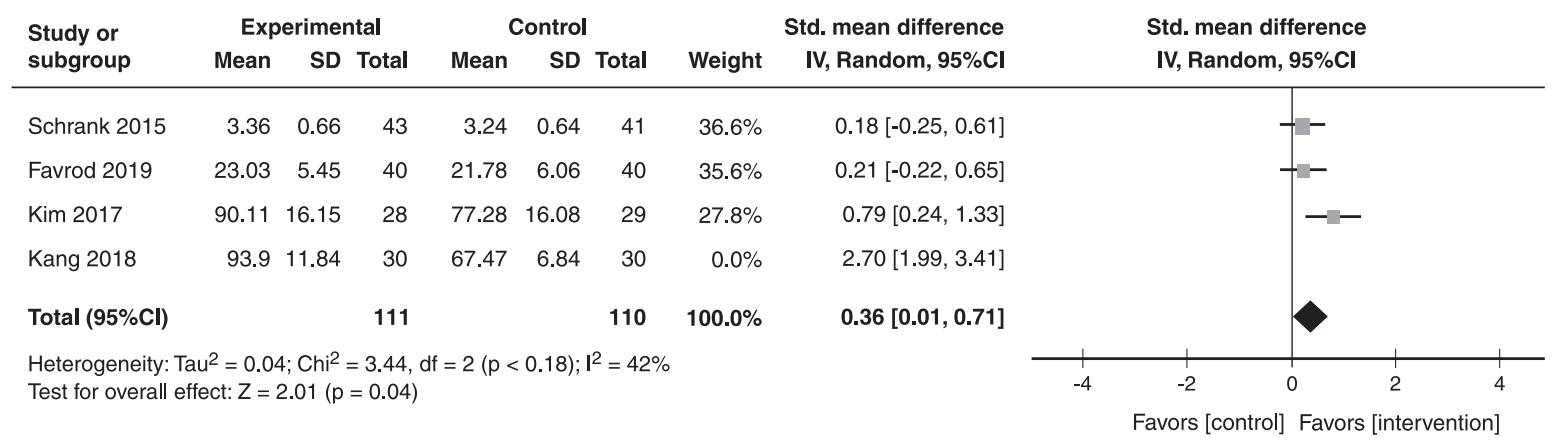

Figure 3 Forest plot of sensitivity analysis excluding Kang et al. ${ }^{22} 95 \% \mathrm{Cl}=95 \%$ confidence interval; $\mathrm{df}=$ degrees of freedom; IV = inverse variance; SD = standard deviation.

practice for these patients, due to the risk of triggering positive psychotic symptoms. ${ }^{32}$

Despite increasing research interest in the concept of well-being in recent years, it remains ill-defined. In the studies identified in this review, five different scales were used as outcome measures to assess well-being: the Scales of Psychological Well Being (SPWB), ${ }^{33}$ Quality of Life Scale (QLS), ${ }^{34}$ Satisfaction with Life Scale (SWLS), ${ }^{35}$ Warwick-Edinburgh Mental Well-being Scale (WEMWBS), ${ }^{36}$ and Manchester Short Assessment (MANSA). ${ }^{37}$ These scales covered a wide variety of conceptual contexts, with the greatest overlap found between the concepts of well-being and quality of life. There was no agreed definition or structure for well-being, and the authors generally did not state why they chose a specific scale. The review by Schrank et al. ${ }^{13}$ identified, in the 28 studies included in the analysis, 20 different scales for assessing well-being. This conceptual gap reflects academic debates about the extent to which well-being, quality of life, and other related constructs can overlap. Therefore, caution is recommended regarding the comparability between the results of the included studies, since the possibility of comparing well-being assessments carried out by different scales is not yet well established.

The studies selected for the quantitative analysis used more than one scale to assess elements of well-being. Therefore, the decision was made to select those most representative. Schrank et al. ${ }^{19}$ used WEMWBS scores; Kim \& $\mathrm{Na},{ }^{21}$ the Connor-Davidson Resilience Scale; Favrod et al., ${ }^{6}$ the Savoring Beliefs Inventory (SBI); and Kang, ${ }^{22}$ a self-esteem scale.

The ACIPS social bonding factor was chosen as the measure more closely related to well-being in the Favrod et al. $^{6}$ trial, since several well-being and positive psychology measures focus on social and inter-relational competences.

Despite being central to PPT, assessment of character strengths was not present in the selected articles. ${ }^{4}$ The $\mathrm{SBI}$ is a 24-item scale that assesses the ability to obtain pleasure by anticipating positive future events, savoring positive moments in the present, and recalling past positive experiences, with scores ranging from 1 to $7 .^{38}$ This scale was present in the evaluation of five of the nine included studies, ${ }^{3,6,17-19}$ which may suggest its superiority in measuring results related to well-being in positive psychology.
Meta-analytic data from the four studies that included controls supported a small effect size for PPI on wellbeing outcomes. However, the data was highly heterogeneous $\left(I^{2}=92 \%\right)$, requiring caution in interpretation. Every study in this review evaluated the acceptability and feasibility of the proposed PPI. In general, participants who remained until the end of the interventions were satisfied, with a positive assessment of usefulness and viability of the intervention. Dropout rates were low, ranging from $8.75^{2}$ to $16 \% .{ }^{18}$ Therefore, acceptability rates were as expected according to literature, since the average dropout rate for similar interventions is usually $13 \% .^{39}$

Our review has several limitations. First, most of the included publications were conducted as pilot studies (7 out of 9 selected articles), with a small number of patients and using a variety of interventions. ${ }^{40,41}$ Second, only three studies were considered to be of high quality according to the criteria adopted. Third, follow-up periods were short, which was also reflected in the reported results. Since PPI techniques involve changes in the way in which one faces life's challenges, perhaps a longer duration of intervention and/or longer follow-up periods could further elucidate the clinical implications of PPIs.

Another important limitation is the still-imprecise definition of what constitutes well-being: resilience, self-esteem, happiness, and even recovery ${ }^{13}$ have been used in studies interchangeably with this concept. ${ }^{14}$

In conclusion, used as an adjunct to drug treatment, PPIs appear to be effective in increasing outcomes related to well-being and, possibly, reducing negative symptoms in patients with schizophrenia-spectrum disorders, but demonstrate no effect in reducing positive symptoms. Despite these promising results, studies involving well-structured protocols with PPT techniques are necessary to improve research quality, comparability and data homogeneity regarding well-being outcomes and patients with psychotic symptoms.

\section{Disclosure}

The authors report no conflicts of interest.

\section{References}

1 Machado L, Matsumoto L. Psicologia positiva e psiquiatria positiva: a ciência da felicidade na prática clínica. São Paulo: Manole; 2020. 
2 Liberman RP. Recovery from schizophrenia: form follows functioning World Psychiatry. 2012;11:161-2.

3 Meyer PS, Johnson DP, Parks A, Iwanski C, Penn DL. Positive living: a pilot study of group positive psychotherapy for people with schizophrenia. J Posit Psychol. 2012;7:239-48.

4 Seligman ME, Steen TA, Park N, Peterson C. Positive psychology progress: empirical validation of interventions. Am Psychol. 2005;60: 410-21.

5 Schrank B, Riches S, Coggins T, Rashid T, Tylee A, Slade M. WELLFOCUS PPT - modified positive psychotherapy to improve well-being in psychosis: study protocol for pilot randomised controlled trial. Trials. 2014;15:203.

6 Favrod J, Nguyen A, Chaix J, Pellet J, Frobert L, Fankhauser C, et al. Improving pleasure and motivation in schizophrenia: a randomized controlled clinical trial. Psychother Psychosom. 2019;88:84-95

7 Owen MJ, Sawa A, Mortensen PB. Schizophrenia. Lancet. 2016;388: 86-97.

8 Pina I, Machado L. Avaliação do bem-estar em pacientes em uso de antipsicóticos atípicos. In: Machado L, Matsumoto L. Psicologia positiva e psiquiatria positiva: a ciência da felicidade na prática clínica. São Paulo: Manole; 2020. p. 260-9.

9 Moher D, Shamseer L, Clarke M, Ghersi D, Liberati A, Petticrew M, et al. Preferred reporting items for systematic review and metaanalysis protocols (PRISMA-P) 2015 statement. Syst Rev. 2015;4:1.

10 World Health Organization. International Classification of Diseases, 11th revision (ICD-11). Geneva: WHO; 2019.

11 American Psychiatric Association. Diagnostic and Statistical Manual of Mental Disorders, Fourth Edition, Text Revision (DSM-IV-TR). Arlington: American Psychiatric Publishing; 2000.

12 American Psychiatric Association. Diagnostic and Statistical Manual of Mental Disorders, Fifth Edition (DSM-5). Arlington: American Psychiatric Publishing; 2013.

13 Schrank B, Bird V, Tylee A, Coggins T, Rashid T, Slade M. Conceptualising and measuring the well-being of people with psychosis: systematic review and narrative synthesis. Soc Sci Med. 2013;92: 9-21.

14 Valiente C, Espinosa R, Trucharte A, Nieto J, Martínez-Prado L. The challenge of well-being and quality of life: a meta-analysis of psychological interventions in schizophrenia. Schizophr Res. 2019;208: 16-24.

15 DerSimonian R, Laird N. Meta-analysis in clinical trials. Control Clin Trials. 1986;7:177-88.

16 Higgins JP, Thompson SG, Deeks JJ, Altman DG. Measuring inconsistency in meta-analyses. BMJ. 2003;327:557-60.

17 Johnson DP, Penn DL, Fredrickson BL, Kring AM, Meyer PS, Catalino LI, et al. A pilot study of loving-kindness meditation for the negative symptoms of schizophrenia. Schizophr Res. 2011;129: 137-40.

18 Favrod J, Nguyen A, Fankhauser C, Ismailaj A, Hasler JD, Ringuet A, et al. Positive emotions program for schizophrenia (PEPS): a pilot intervention to reduce anhedonia and apathy. BMC Psychiatry. 2015;15:231.

19 Schrank B, Brownell T, Jakaite Z, Larkin C, Pesola F, Riches S, et al. Evaluation of a positive psychotherapy group intervention for people with psychosis: pilot randomised controlled trial. Epidemiol Psychiatr Sci. 2015;25:235-46.

20 Meyer-Kalos PS, Ludwig KA, Gaylord S, Perkins DO, Grewen K, Palsson OS, et al. Enhancing stress reactivity and wellbeing in early schizophrenia: a pilot study of individual coping awareness therapy (I-CAT). Schizophr Res. 2018;201:413-4.
$21 \mathrm{Kim} \mathrm{J}, \mathrm{Na} \mathrm{H}$. [Effects of a positive psychotherapy program on positive affect, interpersonal relations, resilience, and mental health recovery in community-dwelling people with schizophrenia]. J Korean Acad Nurs. 2017;47:638-50.

22 Kang SJ, Ko SH, Kim JY, Kim SR. Effects of a mental fitness positive psychology intervention program on inpatients with schizophrenia in South Korea: a feasibility study. Perspect Psychiatr Care. 2020;56: 6-13.

23 Lim MH, Gleeson JF, Rodebaugh TL, Eres R, Long KM, Casey K, et al. A pilot digital intervention targeting loneliness in young people with psychosis. Soc Psychiatry Psychiatr Epidemiol. 2020;55:877-89.

24 Browne J, Estroff SE, Ludwig K, Merritt C, Meyer-Kalos P, Mueser $\mathrm{KT}$, et al. Character strengths of individuals with first episode psychosis in individual resiliency training. Schizophr Res. 2018;195: 448-54.

25 Riches S, Schrank B, Rashid T, Slade M. WELLFOCUS PPT: modifying positive psychotherapy for psychosis. Psychotherapy (Chic). 2016;53:68-77

26 Nguyen A, Frobert L, McCluskey I, Golay P, Bonsack C, Favrod J. Development of the positive emotions program for schizophrenia: an intervention to improve pleasure and motivation in schizophrenia. Front Psychiatry. 2016;7:13.

27 Kay SR, Fiszbein A, Opler LA. The positive and negative syndrome scale (PANSS) for schizophrenia. Schizophr Bull. 1987;13:261-76.

28 Overall JE, Gorham DR. The brief psychiatric rating scale. Psychol Rep. 106210:799-812.

29 Jeste DV. A fulfilling year of APA presidency: from DSM-5 to positive psychiatry. Am J Psychiatry. 2013;170:1102-5.

30 Brown KW, Ryan RM. The benefits of being present: mindfulness and its role in psychological well-being. J Pers Soc Psychol. 2003;84: 822-48.

31 Tai S, Turkington $\mathrm{D}$. The evolution of cognitive behavior therapy for schizophrenia: current practice and recent developments. Schizophr Bull. 2009;35:865-73.

32 Lustyk MK, Chawla N, Nolan RS, Marlatt GA. Mindfulness meditation research: issues of participant screening, safety procedures, and researcher training. Adv Mind Body Med. 2009;24:20-30.

33 Ryff CD. Happiness is everything, or is it? Explorations on the meaning of psychological well-being. J Pers Soc Psychol. 1989;57: 1069-81.

34 Heinrichs DW, Hanlon TE, Carpenter WT Jr. The quality of life scale: an instrument for rating the schizophrenic deficit syndrome. Schizophr Bull. 1984;10:388-98.

35 Diener E, Emmons RA, Larsen RJ, Griffin S. The satisfaction with life scale. J Pers Assess. 1985;49:71-5.

36 Tennant R, Hiller L, Fishwick R, Platt S, Joseph S, Weich S, et al. The Warwick-Edinburgh mental well-being scale (WEMWBS): development and UK validation. Health Qual Life Outcomes. 2007;5:63.

37 Priebe S, Huxley P, Knight S, Evans S. Application and results of the Manchester short assessment of quality of life (Mansa). Int J Soc Psychiatry. 1999;45:7-12.

38 Bryant FB. Savoring beliefs inventory (SBI): a scale for measuring beliefs about savouring. J Ment Health. 2003;12:175-96.

39 Villeneuve K, Potvin S, Lesage A, Nicole L. Meta-analysis of rates of drop-out from psychosocial treatment among persons with schizophrenia spectrum disorder. Schizophr Res. 2010;121:266-70.

40 Rashid T. Positive interventions in clinical practice. J Clin Psychol. 2009;65:461-6.

41 Rashid T, Seligman M. Psicoterapia positiva: manual do terapeuta. Porto Alegre: Artmed; 2019. 


\title{
Corrigendum
}

\author{
http://dx.doi.org/10.1590/1516-4446-2021-0030
}

We hereby inform that a correction is needed in the article titled "Positive psychology interventions to improve well-being and symptoms in people on the schizophrenia spectrum: a systematic review and meta-analysis" (http://dx.doi.org/ 10.1590/1516-4446-2020-1164), by Pina et al., published in the Brazilian Journal of Psychiatry in December 2020 in ahead of print mode. The data from Shrank et al. 2015 (ref. 19 in the article) were mistakenly included in the metanalysis with values reported as mean and standard error (SE), rather than the correct mean and standard deviation (SD). Updating the values from SE to SD resulted in considerably reduced pooled effect sizes compared to the original metanalysis. Nonetheless, as discussed in the review, the results still favor positive psychology interventions for people in the schizophrenia spectrum, albeit by a very slight margin. Below we reproduce the revised, correct versions of the forest plots shown in Figures 2 and 3.

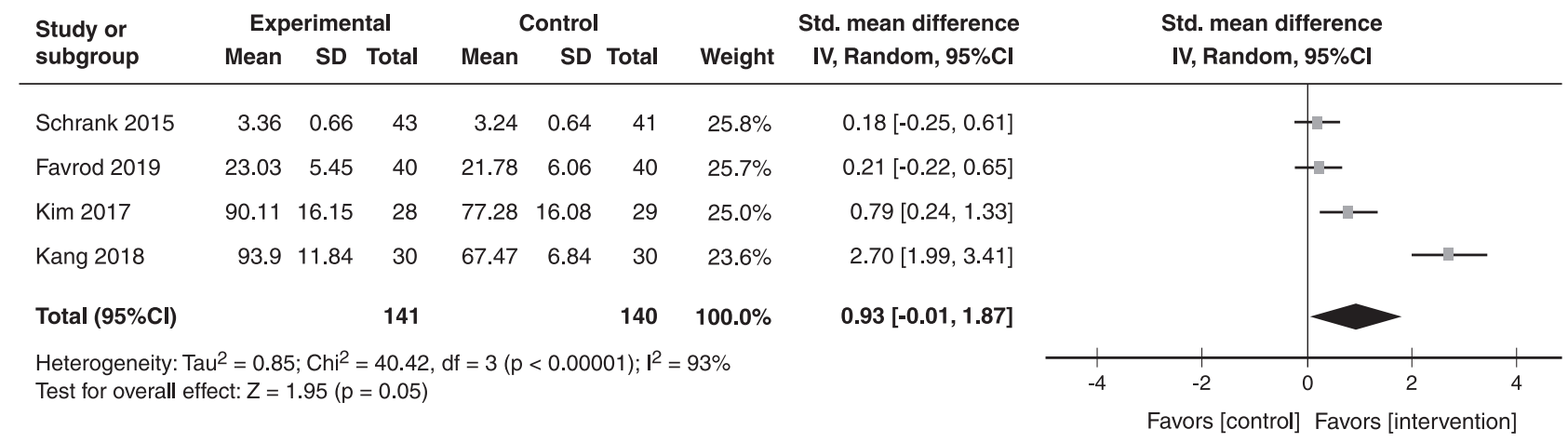

Figure 2 Forest plot and extent of the effects of primary outcomes on well-being and quality of life. $95 \% \mathrm{Cl}=95 \%$ confidence interval; $\mathrm{df}=$ degrees of freedom; IV = inverse variance; SD = standard deviation.

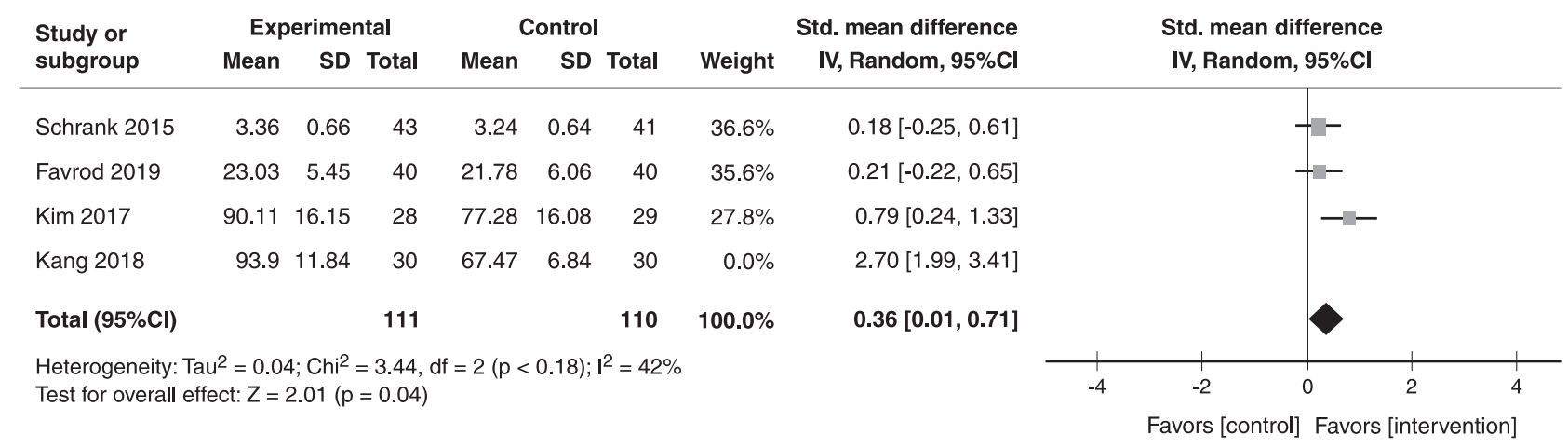

Figure 3 Forest plot of sensitivity analysis excluding Kang et al. ${ }^{22} 95 \% \mathrm{Cl}=95 \%$ confidence interval; df $=$ degrees of freedom; IV = inverse variance; SD = standard deviation. 